\title{
A Heuristic for Fault-Tolerance Provisioning in Multi-Radio Hybrid Wireless-Optical Broadband Access Network
}

\author{
Gabriela Schütz, Noélia S. C. Correia* \\ University of Algarve- Institute of Engineering, Campus da Penha, 8005-139 Faro, Portugal \\ * Center for Electronic, Optoelectronic and Telecommunications \\ University of Algarve- Faculty of Science and Technology, Campus de Gambelas, 8005-139 Faro, Portugal \\ Tel: (+351) 289 800900,Fax: (+351) 289888405 , e-mails:gschutz@ualg.pt
}

\begin{abstract}
The wireless-optical broadband-access network (WOBAN) architecture has been proposed as a flexible and costeffective solution for future access networks. However, for WOBANs to provide geographically continuous wireless coverage it is necessary to integrate fault-tolerance in the design of such networks. In this paper we develop a heuristic algorithm to solve the problem of planning a wireless and optical fault-tolerant multi-radio WOBAN, considering any fault-tolerance scenario (e.g. some nodes/links/region only), while making an efficient reuse of spectrum.
\end{abstract}

Keywords: heuristic, local search, fault-tolerance, channel assignment, hybrid wireless-optical networks.

\section{INTRODUCTION}

The ever-increasing demand for bandwidth in access networks is accelerating research on cost-effective infrastructures and optical-wireless combinations are seen as promising approaches. Wireless-optical broadbandaccess network (WOBAN) architectures provide a flexible and cost-effective solution where fiber is provided as far as possible from the central office ( $\mathrm{CO}$ ) and then wireless access is provided at the front end. Because of such excellent compromise WOBANs are being deployed as municipal access solution [1].

For WOBANs to provide geographically continuous wireless coverage it becomes necessary to integrate fault-tolerance in such networks. The radio band used in wireless networking transmissions (e.g. WiFi) may be common to other systems, due to the use of unlicensed spectrum, leading to the disruption of connections or throughput decrease on all devices within the range. WOBANs should be prepared to avoid such service breaks. This paper proposes a heuristic approach for a joint wireless and optical fault-tolerance planning in multi-radio WOBANs, where mesh routers are equipped with multiple radios that can be tuned to nonoverlapping channels.

The rest of this paper is organized as follows. Section 2 provides an overview on WOBAN and faulttolerance. Section 3 provides a mathematical formalization of the problem as well as some of its properties. Section 4 describes the proposed heuristic while Section 5 analyses results. Section 6 concludes the article.

\section{SURVIVABILITY IN WOBANS}

At the front end a WOBAN consists of a multi-hop wireless mesh network while at the back end an optical access network provides connection to the Internet [1]. At the back end the dominant technology is the passive optical network (PON) having optical line terminals (OLTs), located at the CO, and optical network units (ONUs) to provide connection to wireless gateway routers. Regarding the wireless infrastructure, standard WiFi or WiMAX technology can be used for wireless mesh connectivity. Mesh wireless routers provide multihop connectivity for user traffic delivery toward a few wireless gateway routers that are connected to the ONUs of the optical back end. An ONU can drive multiple gateways.

In WOBANs two fault-tolerance levels must be considered: i) wireless fault-tolerance; ii) optical faulttolerance. In optical fault-tolerance multiple back end failures may exist: i) Gateway, with traffic being redirected to any 'alive' gateway in the network; ii) ONU, meaning that one or multiple gateways will fail and traffic must be redirected to gateways connected to 'alive' ONUs; iii) OLT, meaning that all ONUs connected to the OLT will fail and traffic must be redirected to gateways connected to 'alive' ONUs and 'alive' OLTs. According to the optical fault-tolerance level considered, risk groups (RGs) are defined for gateways, where two gateways belong to the same RG if they may fail at the same time.

The adopted survivability approach assumes precalculated primary and backup routes for every mesh wireless router using the shortest path. Backup routes are failure dependent, meaning that a backup route is activated for a specific link failure of the primary route it protects. Optical fault-tolerance is provided since primary and backup routes use different gateways (easily extended to other fault-tolerance scenarios), while wireless fault-tolerance is ensured by proper channel assignment to radios used by primary and backup routes. This must take into consideration the matrix $I$ that summarizes dependence among feasible wireless links, as in [2]. That is, $I\left[l, l^{\prime}\right]=1$ if $l$ can not transmit, on the same channel, simultaneously with $l^{\prime}$ (not independent). We also define a matrix $I_{r}$ for router dependence, meaning that $I_{r}[u, v]=1$ if $u$ interferes with $v$. The only 
known previous work on WOBAN fault-tolerance is [3] where an alternative path is provided to 'alive' gateways after failure and no integrated wireless and optical fault-tolerance is performed. Here we provide such integrated fault-tolerance together with channel assignment to multi-radio routers using failure dependent backup routes, not previously assumed in the literature.

\section{WOBAN'S SURVIVABILITY PROBLEM}

\subsection{Problem Formulation}

Let us model the network using a directed graph $G=(V, E)$, where $V$ is the set of nodes (routers), each equipped with one or more interface cards (referred here as radios), and $E$ is the set of feasible transmission links. For simplicity the transmission and interference ranges are considered here to be the same for all radios inside a specific node (the work is easily extended to consider different ranges). The subset of routers aggregating local user traffic, and responsible for injecting the user packets into the wireless mesh of the WOBAN, is denoted by $V_{A} \subset V$. The subset of mesh routers acting as gateways, and attached to an ONU, is denoted by $V_{G} \subset V$. The set of available channels per radio is denoted by $C$. The total number of radios is denoted by $R$. A lower bound for $R$ will be $R_{L B}=2\left|V_{A}\right|+\left|V_{G}\right|$ if backup routes are necessary for all the primary routes. The maximum channel transmission rate is normalized as a unit constant and the local traffic demand at any node $u$ is defined proportionally and denoted by $D_{u}$.

Let us define $P$ as the set of precomputed primary routes and $B^{p}$ as the set of precomputed backup routes protecting a specific primary route $p \in P$. Precomputed routes imply that connected series of links associated with routes are known a priori. A specific primary route included in $P$ is denoted by $p$ while a backup route included in $B^{p}$ is denoted by $b$. Every route $p \in P$ or $b \in B^{p}$ can be defined as a connected series of links, written as $p: s(p) \in V_{A} \wedge d(p) \in V_{G}$ and $b: s(b) \in V_{A} \wedge d(b) \in V_{G}$. We define $E^{p}$ and $E_{b}^{p}$ as the set of links used by primary route $p$ and backup route $b$, respectively. In addition we define $E^{p, u}$ and $E_{b, u}^{p}$ as the set of links used by primary route $p$ and backup route $b$, respectively, having router $u \in V$ as its source or destination.

Let $\tau_{c, l}^{p}$ be a binary variable indicating if the traffic of $p \in P$ flows through channel $c \in C$ at link $l \in E^{p}$. Let also $\sigma_{c, l}^{p, b}$ be a binary variable indicating if the backup route $b \in B^{p}$, protecting primary route $p \in P$ against the failure of the link emanating from $s(b)$, flows through channel $c \in C$ at link $l \in E_{b}^{p}$. Let $\rho_{u}^{c}$ be a binary variable indicating if the node $u \in V$ transmits or receives through channel $c \in C$. The following formulation allows any fault-tolerance scenario to be implemented (fault-tolerance may be provided to some nodes/links/regions only):

$$
\min _{l \in E, c \in C}\left(\alpha_{c, l}+\beta_{c, l}\right) \text {, }
$$

where $\quad \alpha_{c, l}=\sum_{p \in P} \sum_{l^{\prime} \in E^{p}: I\left(l^{\prime}, l\right)=1} \tau_{c, l^{\prime}}^{p} \times D_{s(p)}$ and $\beta_{c, l}=\max _{l^{\prime} \in E, c^{\prime} \in C} \sum_{p \in P: \tau_{c^{\prime}, l^{\prime}}=1} \sum_{l^{\prime} \in E_{b}^{p}: I\left(l^{\prime}, l\right)=1} \sigma_{c, l^{\prime}}^{p, b} \times D_{s(p)}$. For traffic to flow through channels of primary and backup routes the objective is subject to $\sum_{c \in C} \tau_{c, l}^{p}=1, \forall p \in P, \forall l \in E^{p} \quad$ and $\sum_{c \in C} k_{c, l}^{p, b}=1, \forall p \in P, \forall b \in B^{p}, \forall l \in E_{b}^{p}$. To protect the wireless network against radio failures and interferences, a backup route can not use the same channel, as the link, $l$, of the primary route it protects, at links that are not independent of $l: \sum_{l^{\prime} \in E_{b}^{p}: I\left(l^{\prime}, l\right)=1} \sigma_{c, l^{\prime}}^{p, b} \leq\left(1-\tau_{c, l}^{p}\right) \times\left|E_{b}^{p}\right|, \forall p \in P, \forall c \in C, \forall l \in E, \quad b \in B^{p}: s(b)=s(l)$. To force $\rho_{u}^{c}$ to be one if at least one primary or backup route uses a particular channel, $c$, at some specific node $u$ :

$$
\rho_{u}^{c} \geq\left(\sum_{p \in P} \sum_{l \in E^{p, u}} \tau_{c, l}^{p}+\sum_{p \in P} \sum_{b \in B^{p}} \sum_{l \in E_{b, u}^{p}} \sigma_{c, l}^{p, b}\right) /\left(\sum_{p \in P}\left|E^{p, u}\right|+\sum_{p \in P} \sum_{b \in B^{p}}\left|E_{b, u}^{p}\right|\right), \forall c \in C, \forall u \in V .
$$

The number of radios is limited by $\sum_{u \in V} \sum_{c \in C} \rho_{c}^{u} \leq R$. Variables $\tau_{c, l}^{p}, \rho_{u}^{c}, \sigma_{c, l}^{p, b}$ are binary and $\alpha_{c, l}, \beta_{c, l}$ are float.

\subsection{Problem Properties}

Considering single radio gateway routers, $g_{i}$, dual-radio mesh routers, $u, v \in V_{A}$, and any precomputed primary and backup routes, we enunciate the following propositions:

Proposition 1: If gateway routers $g_{i_{1}}$ and $g_{i_{2}}$ interfere, then they must have their receivers tuned to different channels. Mesh routers simultaneously adjacent to $g_{i_{1}}$ and $g_{i_{2}}$ must have their radios assigned to those two different channels. 
Proof: $I_{r}\left[g_{i_{1}}, g_{i_{2}}\right]=1 \Rightarrow \exists l, l^{\prime} \in E: I\left[l, l^{\prime}\right]=1$; let $l=\left(u, g_{i_{1}}\right) \in p$, for a $p \in P$, and $l^{\prime}=\left(v, g_{i_{2}}\right) \in b$, for $b \in B^{p}$, then to provide fault-tolerance $\tau_{c_{j_{1}}, l}^{p}=1 \wedge \sigma_{c_{j_{2}}, l^{\prime}}^{p, b}=1$ for channels $c_{j_{1}}, c_{j_{2}}: c_{j_{1}} \neq c_{j_{2}}$, which implies that $\rho_{g_{i_{1}}}^{c_{j_{1}}}=\rho_{g_{i_{2}}}^{c_{j_{2}}}=1$ and $\rho_{u}^{c_{j_{1}}}=\rho_{v}^{c_{j_{2}}}=1$ ( if $u=v$ then $\rho_{u}^{c_{j_{1}}}=\rho_{u}^{c_{j_{2}}}=1$ ).

Proposition 2: If two adjacent mesh routers, $u$ and $v$, are also adjacent to a gateway, $g_{i}$, then they must have the same channel assignment in their two radios.

Proof: let $\rho_{g_{i}}^{c_{j_{1}}}=1$ then $\rho_{u}^{c_{j_{1}}}=\rho_{v}^{c_{j_{1}}}=1$; let $l=\left(u, g_{i}\right) \in p$, for a $p \in P$, and $l^{\prime}=(u, v) \in b$, for $b \in B^{p}$, then $\tau_{c_{j_{1}}, l}^{p}=1 \wedge \sigma_{c_{j 1}, l^{\prime}}^{p, b}=0, \sigma_{c_{j 2}, l^{\prime}}^{p, b}=1, c_{j_{1}} \neq c_{j_{2}}$, and consequently $\rho_{u}^{c_{j 2}}=\rho_{v}^{c_{j 2}}=1$.

As a consequence of these propositions we conclude that: if gateway routers $g_{i_{1}}$ and $g_{i_{2}}$ interfere, router $u$ is adjacent to $g_{i_{1}}$ and router $v$ is adjacent simultaneously to $g_{i_{1}}$ and $g_{i_{2}}$, then router $u$ must have the same channel assignment of router $v$.

Proposition 3: If gateway routers $g_{i_{1}}, g_{i_{2}}$ and $g_{i_{3}}$ interfere, and a router $u$ is adjacent to two of them and no more than two links join $u$ to the other gateway, then there is no feasible solution.

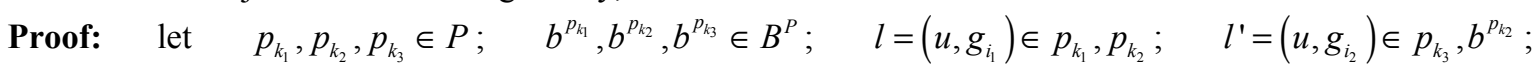
$l^{\prime \prime}=\left(v, g_{i_{3}}\right) \in b^{p_{k_{1}}}, b^{p_{k_{3}}} ; l^{\prime \prime}=(u, v) \in b^{p_{k_{1}}}, b^{p_{k_{3}}}$ (interfering links). To provide fault-tolerance $\rho_{g_{i_{1}}}^{c_{j_{1}}}=\rho_{g_{i_{2}}}^{c_{j_{2}}}=\rho_{g_{i_{3}}}^{c_{j_{3}}}=1$, with different $c_{j_{1}}, c_{j_{2}}, c_{j_{3}}$, then $\rho_{u}^{c_{j_{1}}}=\rho_{u}^{c_{j_{2}}}=\rho_{v}^{c_{j_{3}}}=1$. As $\rho_{v}^{c_{j_{2}}}=1$ or $\rho_{v}^{c_{j_{1}}}=1$ there is no feasible channel assignment to $l^{\prime \prime \prime} \in b^{p_{k_{3}}}$ or to $l^{\prime \prime \prime} \in b^{p_{k_{1}}}$, respectively, then no feasible solution can be found. Clearly, this result still holds for the particular case when a router is adjacent to three (or more) gateways.

The reasoning behind these propositions provides feasible ways of channel assignment with a glance at the neighbour links and routers, belonging to routes that include the link being addressed for channel assignment.

\section{TWO-PHASE HEURISTIC}

Based on the above formulation and propositions we implement a two-phase heuristic that we shortly describe:

Phase 1 - Construction of a feasible solution for the minimum feasible number of $\operatorname{radios}\left(R=R_{L B}\right)$

(i) reduce size problem applying propositions 1 and 2 to assign channels to gateways, routers and links;

(ii) sequentially assign feasible channels to primary links and then to backup links minimizing, respectively, $\alpha_{c, l}$ and $\beta_{c, l}$ (by condition (2) channel assignment of involved radio routers are implicit);

(iii) calculate $f=\alpha_{c, l}+\beta_{c, l}$ for the channel assignment solution achieved in (i) and (ii) and this is the incumbent solution.

Phase 2 - Local search to obtain solutions for $R=R_{L B}+1$ to $R=R_{L B}+m$ radios (with $m \in \mathbb{N}$ defined a priori)

(i) consider routers $u, v \in V: u=s(l) \wedge v=d(l)$, where $l$ is the most loaded link in the previous solution and obtain $N=\{$ adjacent routers of $u\} \cup\{$ adjacent routers of $v\}$;

(ii) sequentially add a radio for each of the $N$ routers and reassign feasible channels to primary and backup neighbour links of the router being addressed (consequently perform routers channel assignment);

(iii) retain the best solution, $f$, achieved in (ii) and if it is better then the incumbent solution update this one;

(iv) go to (i).

\section{ANALYSIS OF RESULTS}

In this section we analyse the results of the joint wireless and optical survivability planning two-phase heuristic. Results were obtained for the SFNet, a WOBAN in San Francisco discussed in [4]. The computational study was carried out on a $3.0 \mathrm{GHz}$ PentiumIV processor with $1 \mathrm{~GB}$ RAM. The heuristic code was written in C.

Every traffic demand is set to $0.5\left(D_{u}=0.5, \forall u \in V_{A}\right)$, assuming that the maximum channel transmission rate is normalized as a unit constant. Precomputed primary routes and link failure dependent backup routes, using the shortest path and having a gateway router as destination, are given as input. Gateways are at different risk groups meaning that the only requirement for optical protection is that primary and backup routes must be connected to different gateways. The available number of independent channels in a router, $|C|$, is set to 3 , and $m$ is set to 10 .

\subsection{Load Measurement}

The plots in Fig. 1 show the load of the most overloaded link channel for the heuristic solution. When no faulttolerance is provided the load of the most overloaded link channel is 2.5 , also plotted in the figure. This value does not change as the number of radios increases because the spectrum becomes exhausted, after a certain 
number of radios (around 30 radios), when no fault-tolerance is provided. In this case a single radio per node is necessary, 25 radios in total. For fault-tolerance to be provided, at least 2 radios must exist at mesh routers while gateways are allowed to have a single radio. The assessment of the quality of the solutions produced by the heuristic was made by comparison with the optimal solution (determined using formulation in section 3.1), also plotted in Fig. 1. The heuristic efficiency is good, considering that the heuristic approach took only 0.047 seconds of computational execution while the optimal solution required hours of computation time; and that two solutions differ for a multiple of 0.5 as router demands are not allowed to be split.

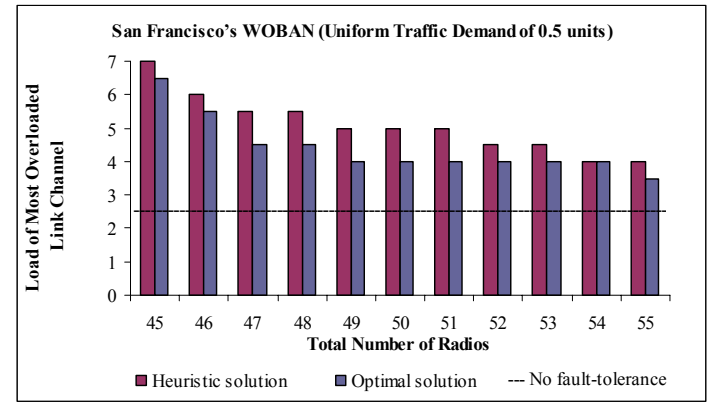

Figure 1. SFNet's most overloaded link channel.

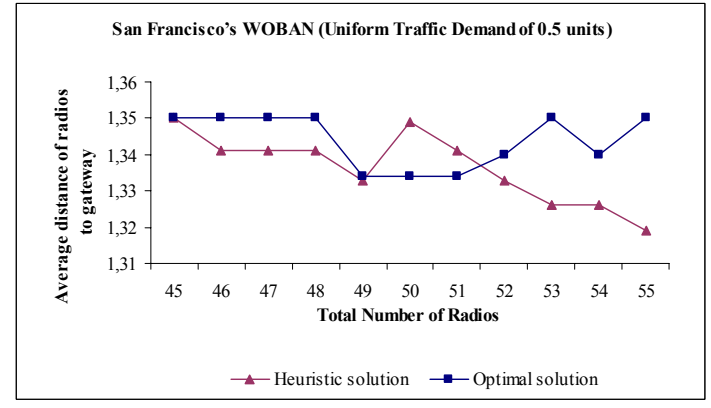

Figure 2. SFNet's average distance

The values indicate that a small increase in the number of radios can decrease the total capacity required to provide fault-tolerance, even with only a near-optimal solution as the one produced with this heuristic.

\subsection{Radio Distribution}

The average distance from every radio to the nearest gateway, $\bar{h}$, as the total number of radios available in the network increases is plotted in Fig. 2. The $\bar{h}$ value can be determined by

$$
\bar{h}=\left(\sum_{u \in V_{A}} h_{u} \sum_{c \in C} \rho_{u}^{c}\right) / \sum_{u \in V_{A}} \sum_{c \in C} \rho_{u}^{c},
$$

where $h_{u}$ is the minimum number of hops from node $u$ to any gateway. The optimal radio distribution at the network is found when the average distance reaches its minimum.

As it can be seen in Fig. 2 the average distance from radios to gateways starts decreasing, as the set of available radios increases. This means that the gateways and nodes near the gateways, being more congested are stronger candidates to receive extra radios. In what concerns to the heuristic, the optimal distribution of radios is achieved for 55 radios. The increase in the average distance between 49 and 51 radios reflects the non improvement in capacity usage, shown in Fig. 1. We observe a different behaviour of radio distribution obtained with the exact algorithm, from 49 to 55 radios.

\section{CONCLUSIONS}

In this paper we develop and test a heuristic approach to solve integrated routing and channel assignment in multi-radio WOBANs aimed at obtaining a fault-tolerant network. The heuristic efficiency is measured comparing with exact solutions and it proves well enough as a near-optimal real-time solution is obtained. The analysis of results indicate that while in multi-radio WOBANs with no fault-tolerance provisioning the spectrum reutilization becomes difficult, due to spectrum exhaustion, this is possible when fault-tolerance is provided using our heuristic approach.

\section{ACKNOWLEDGMENTS}

This work was supported by the Foundation for Science and Technology from Portugal within CEOT (Center for Electronic, Optoelectronic and Telecommunications) and by the project PTDC/EEA-TEL/71678/2006.

\section{REFERENCES}

[1] S. Sarkar et al.: A novel delay-aware routing algorithm (DARA) for a hybrid wireless-optical broadband access network (WOBAN), IEEE Network, vol. 22, no. 3, pp. 20-28, May/June 2008.

[2] J. Zhang et al.: Joint routing and scheduling in multi-radio multi-channel multi-hop wireless networks, International Conference on Broadband Networks, vol. 1, pp. 631-640, Oct. 2005.

[3] S. Sarkar et al:: RADAR: Risk-and-Delay Aware Routing Algorithm in a hybrid wireless-optical broadband access network (WOBAN), IEEE/OSA OFC, Mar. 2007.

[4] S. Sarkar et al.: DARA: Delay-Aware Routing Algorithm in a hybrid wireless-optical broadband access network (WOBAN), IEEE ICC, pp. 2480-2484, June 2007. 